\title{
EDUCAÇÃO EM SAÚDE COM PESSOAS USUÁRIAS DE ÁLCOOL E OUTRAS DROGAS
}

\author{
Roselma Lucchese ${ }^{1}$, Fernanda Karla Nascimento ${ }^{2}$, Ivânia Vera ${ }^{1}$, Rodrigo Lopes de Felipe ${ }^{3}$, Inaína Lara \\ Fernandes ${ }^{4}$, Alexandre de Assis Bueno ${ }^{5}$
}

\begin{abstract}
RESUMO: O objetivo foi analisar a constituição de conhecimento entre pessoas em reabilitação do uso e abuso de drogas a partir de grupos de educação em saúde. Pesquisa convergente assistencial com indivíduos internados em comunidade terapêutica. Utilizou-se um questionário semiestruturado e autoaplicável para analisar o nível de conhecimento pré e pós-intervenção. A coleta ocorreu entre agosto de 2013 e fevereiro de 2014. Participaram do estudo 28 homens usuários de múltiplas substâncias psicoativas. Houve aumento nas definições de efeitos, reconhecimento de estratégias de reabilitação, classificação e ação de substâncias pelos participantes. A análise qualitativa revelou diferença na qualidade e na profundidade das respostas pré e pós-intervenção. A educação em saúde edificou o conhecimento dos saberes primários quanto ao uso abusivo de álcool e outras drogas, e sua correlação com o próprio comportamento, além de sua contextualização e reflexão acerca da reabilitação. DESCRITORES: Usuários de drogas; Educação em saúde; Enfermagem; Saúde pública; Promoção da saúde.
\end{abstract}

\section{HEALTH EDUCATION WITH USERS OF ALCOHOL AND OTHER DRUGS}

ABSTRACT: The objective of this study was to analyze the acquisition of knowledge among people undergoing rehabilitation for drug abuse in health education groups. Convergent-care research was developed with individuals admitted in therapeutic communities. A semi-structured and self-administered questionnaire for analyzing the level of pre- and post-intervention knowledge was used. Data were collected between August 2013 and February 2014. Twenty-eight male users of multiple psychoactive substances participated in the study. There was an increase in the definitions of the effects, awareness of rehabilitation strategies, substance classification and action by the participants. Qualitative analysis revealed differences in quality and depth of the pre- and post-intervention answers. Health education raised the understanding on basic knowledge related to abuse of alcohol and other drugs, and its correlation to the behavior, in addition to its contextualization and reflection regarding the rehabilitation DESCRIPTORS: Drug users; Health education; Nursing; Public health; Health promotion.

\section{EDUCACIÓN EN SALUD CON PERSONAS CONSUMIDORAS DE ALCOHOL Y OTRAS DROGAS}

RESUMEN: El objetivo fue analizar la constitución de conocimiento entre personas en rehabilitación de uso y abuso de drogas a partir de grupos de educación en salud. Investigación convergente asistencial con individuos internados en comunidad terapéutica. Se utilizó un cuestionario semiestructurado autoaplicable para analizar el nivel de conocimiento previo y posterior a intervención. Datos recolectados entre agosto de 2013 y febrero de 2014. Participaron del estudio 28 hombres consumidores de múltiples sustancias psicoactivas. Hubo aumento en las definiciones de efectos, reconocimiento de estrategias de rehabilitación, clasificación y acción de sustancias de parte de los participantes. El análisis cualitativo expresó diferencias en la calidad y profundidad de las respuestas previas y posteriores a intervención. La educación en salud edificó el conocimiento de los saberes primarios respecto del uso abusivo de alcohol y otras drogas, y su correlación con el propio comportamiento; además de su contextualización y reflexión acerca de la rehabilitación.

DESCRIPTORES: Consumidores de Drogas; Educación en Salud; Enfermería; Salud Pública; Promoción de la Salud.

${ }^{1}$ Enfermeira. Doutora em Enfermagem. Docente de Enfermagem da Universidade Federal de Goiás. Catalão, GO, Brasil.

${ }^{2}$ Enfermeira. Universidade Federal de Goiás. Catalão, GO, Brasil.

${ }^{3}$ Farmacêutico. Mestre em Ciências Veterinárias, Morfologia e Saúde. Docente de Enfermagem da Universidade Federal de Goiás. Catalão, GO, Brasil.

${ }^{4}$ Enfermeira. Mestranda em Gestão Organizacional. Universidade Federal de Goiás. Catalão, GO, Brasil.

${ }^{5}$ Enfermeiro. Mestre em Enfermagem. Docente de Enfermagem da Universidade Federal de Goiás. Catalão, GO, Brasil.

Autor Correspondente:

Recebido: 08/06/2016

Roselma Lucchese

Universidade Federal de Goiás

Finalizado: 18/11/2016

Av. Lamartine P. de Avelar, 1120 - 75.704-020 - Catalão, GO, Brasil

Email: roselmalucchese@gmail.com 


\section{INTRODUÇÃO}

O uso e o abuso simultâneos de múltiplas drogas, lícitas e ilícitas entre jovens adultos têm preocupado os estudiosos ao considerarem a maior probabilidade de dependência química quando comparada a outros estratos etários. Também se observa aumento deste comportamento em diversos segmentos sociais ${ }^{(1-3)}$. Em relação à saúde, o uso crônico de substâncias psicoativas provoca danos nas dimensões fisiológicas, sociais e econômicas ${ }^{(1,4-6)}$.

O desafio está na constituição de práticas de promoção e prevenção de agravos à saúde desses usuários. Uma das propostas pauta-se na educação em saúde, que favorece a constituição de novos conhecimentos, mudanças de hábitos, atitudes e comportamento, seja no âmbito individual seja no coletivo $^{(7)}$. Em especial para o Enfermeiro, a educação em saúde na área de dependência química é uma provocação ${ }^{(8)}$, uma vez que necessita do engendramento e da articulação de saberes do educador no atendimento à população alvo, com foco na conscientização das consequências do uso abusivo de drogas $^{(9)}$.

Por parte de pessoas que vivem com comorbidade e, em uso abusivo de álcool e outras drogas, avaliam a educação em saúde como um instrumento efetivo na promoção de sua saúde, com ganhos no desempenho dos papéis assumidos na vida ${ }^{(8)}$. Uma vez que esta tecnologia viabiliza a construção coletiva da saúde, da cidadania, da expressão do ser e torna os espaços de discussão relevantes para sua existência ${ }^{(7)}$. Em se tratando de dependência química, é neste âmbito que se possibilita a reflexão sobre um tema discriminado pela sociedade e autoridades. Assim, ilumina-se as decisões sobre si, suas relações e o próprio tratamento ${ }^{(9)}$.

Neste estudo, o interesse foi pela efetivação de educação em saúde para dependentes químicos, com inspiração nos pressupostos da psicoeducação. Que, sumariamente, acompanha clinicamente o indivíduo quanto ao processo saúde-doença, e promove a construção do autoconhecimento, com intuito de melhorar o enfrentamento de situação vivida e estimular a participação ativa em seu tratamento/reabilitação ${ }^{(10-11)}$. O objetivo do estudo foi analisar a constituição de conhecimento entre pessoas em reabilitação do uso e abuso de álcool e outras drogas, a partir de grupos de educação em saúde inspirados na psicoeducação, como contribuição à estruturação de modelos de intervenção no processo de cuidados em enfermagem.

\section{MÉTODO}

Trata-se de uma pesquisa convergente assistencial, com aplicação da tecnologia de educação em saúde inspirada na psicoeducação. Isso requer o envolvimento do pesquisador com as questõesproblema advindas da prática, indicando a produção de conhecimento do saber-fazer assistencial, resultando em impacto e reestruturação no modelo de atenção em enfermagem ${ }^{(12)}$.

Desse modo, houve definição do tema, campo de atuação, sujeitos e métodos de análise. Posteriormente, a fase da perscrutação aprofundou a coleta de dados, com o objetivo de produzir material para a própria pesquisa e fomentar o cuidado de enfermagem, aprimorando-o. Na análise, buscou-se a interpretação dos dados, examinando-os subjetivamente e associando-os às teorias escolhidas para compreensão da realidade e contexto ${ }^{(12)}$.

O estudo foi desenvolvido em uma comunidade terapêutica masculina localizada no sudeste do Estado de Goiás. Os critérios de inclusão: idade $\geq 18$ anos, fazer uso de substâncias psicoativas, estarem internados na comunidade terapêutica no momento da entrevista, responder aos questionários antes e pós-intervenção. Foram excluídos: sujeitos com diagnóstico médico de transtorno mental grave e persistente e aqueles que estavam aparentemente sedados ou em estado de confusão mental, após realização do exame físico do nível de consciência no dia da entrevista. A coleta de dados, desde a fase de perscrutação, ocorreu entre agosto de 2013 e fevereiro de 2014, na comunidade terapêutica.

Para as intervenções grupais, estabeleceu-se o contrato pedagógico-assistencial, e foram realizadas em dois subgrupos, em que os mesmos temas foram discutidos em 8 encontros de 60 minutos de duração cada. Os primeiros 20 minutos eram de exposição do tema e no restante do tempo abria-se o 
espaço dialógico-reflexivo. Os temas estão expressos no Quadro 1.

O questionário semiestruturado autoaplicável continha perfil sociodemográfico do sujeito e questões discursivas referentes a conceito, efeitos e classificação de substâncias psicoativas, bem como estratégias de reabilitação. Esse instrumento permitiu a avaliação da construção de conhecimento a partir dos grupos de educação em saúde. Todo esse processo de intervenção e análise foi pautado nas etapas da pesquisa convergente assistencial (Quadro 1).

Quadro 1 - Síntese das fases do estudo e atividades desenvolvidas na pesquisa convergente assistencial. Sudeste Goiano, GO, Brasil, 2014

\begin{tabular}{|l|l|}
\hline Fase do estudo & Atividade \\
\hline Concepção & $\begin{array}{l}\text { Tema de pesquisa: educação em saúde com pessoas em reabilitação pelo abuso de } \\
\text { álcool e outras drogas }\end{array}$ \\
\hline Instrumentação & $\begin{array}{l}\text { Escolha da tecnologia de grupos de educação e planejamento do modo de } \\
\text { funcionamento dos mesmos }\end{array}$ \\
\hline Perscrutação & $\begin{array}{l}\text { Aplicação de questionários antes/depois } \\
\text { Temas dos grupos: } \\
\text { - Fisiologia do sistema nervoso central } \\
\text { - Conceito, classificação das drogas e efeitos nocivos } \\
\text { - Tipos de substâncias químicas } \\
\text { - Concepção de Reabilitação }\end{array}$ \\
\hline Análise & $\begin{array}{l}\text { Tabulação das respostas do questionário antes/depois, orientada por alteração de } \\
\text { padrão das respostas. } \\
\text { Análise qualitativa de conteúdo: leitura flutuante, desprendimento das UR* } \\
\text { constituição do corpus da pesquisa, codificação, agrupamento e categorização }\end{array}$ \\
\hline Interpretação & $\begin{array}{l}\text { Síntese, teorização e significação dos resultados com sugestão de modelos para a } \\
\text { atenção de enfermagem }\end{array}$ \\
\hline
\end{tabular}

*UR: Unidades de Registro.

As intervenções envolveram uma enfermeira, um farmacêutico e seis graduandos do curso de enfermagem, pesquisadores de campo que já tinham concluído a disciplina de saúde mental. As Unidades de Registro (UR), ou seja, os fragmentos de respostas dos sujeitos foram codificados na letra S, seguida do número de ordem (S1, S2...). Entre parênteses, indicou-se a idade de cada participante e, após, designou-se a resposta 'A' para antes e 'D' para depois da intervenção.

Esta pesquisa integra um projeto matriz que buscou analisar a atenção à saúde de pessoas em uso e abuso de álcool, tabaco e outras drogas, e foi analisado e aprovado em 14 de dezembro de 2014 pelo Comitê de Ética em Pesquisa da Universidade Federal de Goiás, sob parecer $n^{\circ} 926.819$. Todos os sujeitos assinaram o Termo de Consentimento Livre e Esclarecido, conforme preceitos éticos, diretrizes e normas regulamentadoras de pesquisa envolvendo seres humanos dispostos na Resolução $\mathrm{n}^{\circ} 466$, de 12 de dezembro de $2012^{(13)}$.

\section{RESULTADOS}

Participaram dos grupos 39 homens, contudo 11 destes não atenderam ao critério de responder aos questionários antes e pós-intervenção, por altas ou abandonos de tratamento que ocorreram durante a intervenção. Diante do exposto, o estudo constituiu-se de 28 homens internos usuários de múltiplas substâncias psicoativas, sobretudo do tabaco, álcool e alguma droga ilícita. A média de idade foi 32 anos e a maioria tinha o Ensino Fundamental incompleto ( $n=10 / 36 \%)$. Os padrões de respostas quantificadas estão dispostos no Quadro 2 e distribuídas em duas categorias: Conhecimento de base quanto ao uso de substâncias psicoativas e Conhecimento correlacionado/contextualizado. 
Quadro 2 - Categorização e conhecimento do sujeito antes e depois da intervenção em educação em saúde. Sudeste Goiano, GO, Brasil, 2014

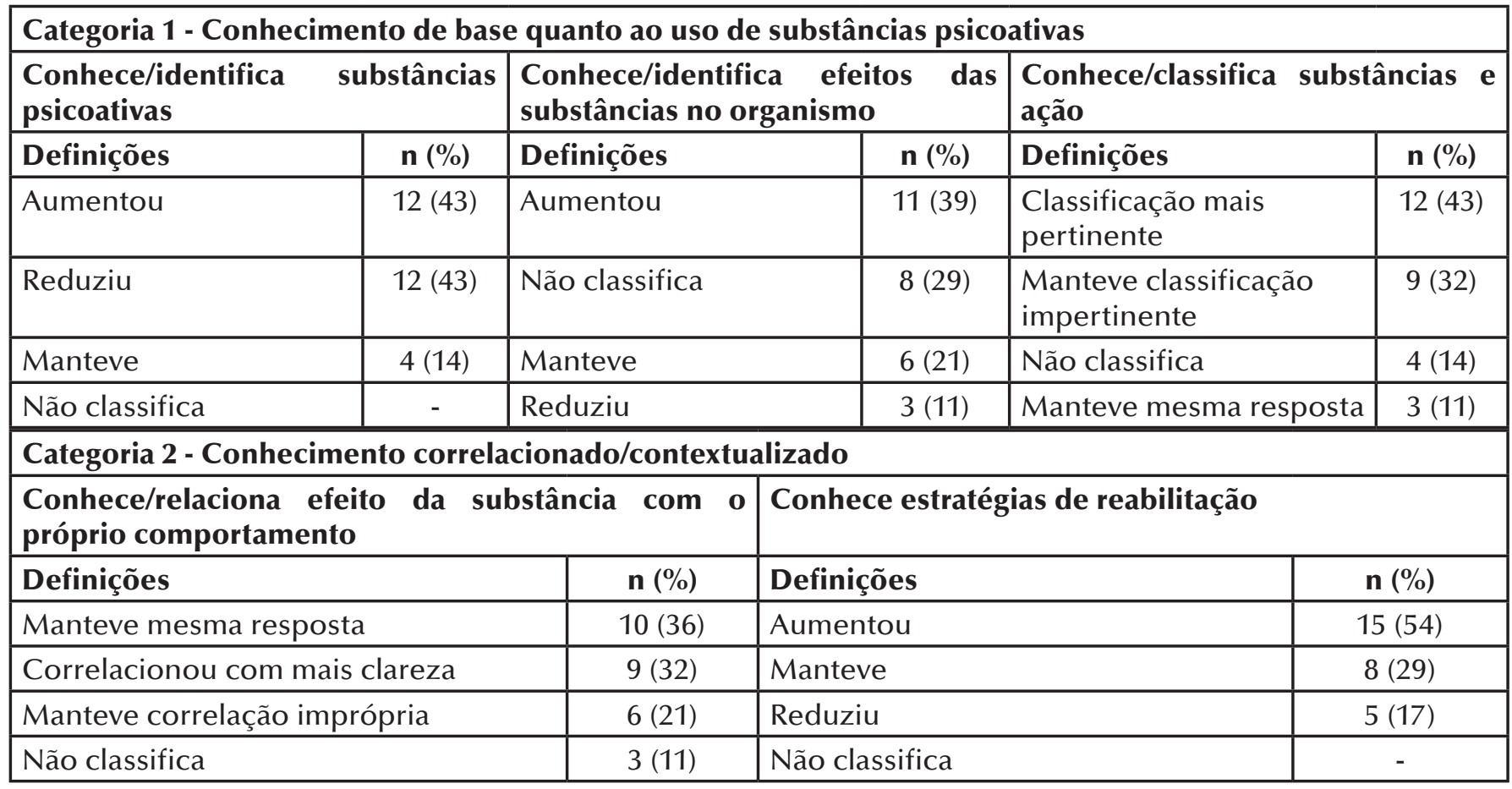

Observa-se igual porcentagem das respostas que reduziram e aumentaram a identificação de substâncias após a intervenção. Quanto ao efeito das substâncias no organismo, de reconhecimento de estratégias de reabilitação, classificação e ação de substâncias, houve aumento das definições. Não houve alteração entre o antes e o depois na maioria das respostas sobre relacionar o efeito do álcool e/ou droga com o próprio comportamento.

A avaliação qualitativa da intervenção em saúde orientou-se pela análise quantitativa, direcionando o agrupamento das UR para dois aspectos avaliados: os que mantiveram ou reduziram o padrão de resposta, e aqueles que apresentaram respostas com conteúdo ampliado ou contextualizado. Abaixo estão algumas respostas obtidas por meio dos depoimentos dos sujeitos, antes e depois da intervenção.

\section{Categoria 1 - Conhecimento de base quanto ao uso de substâncias psicoativas}

Maconha, merla, crack, tíner, cola, lança-perfume, LSD, ecstasy, álcool. ( $\left.{ }^{*} S 7^{* *}(27)^{* * *} A\right)$. Maconha, $L S D$, cocaína, merla, ecstasy, solventes. $\left({ }^{*} S 7^{* *}(27)^{* * * *} D\right)$

Maconha, merla, crack, cocaína, heroína, LSD, ecstasy, álcool e o pior de todos, o cigarro $\left.{ }^{*} S 10^{* *}(38)^{* * *} A\right)$. Tabaco, LSD, cocaína, anfetamina, heroína, ecstasy, álcool. (S10(38)D)

Maconha, cocaína, crack, haxixe, heroína, lança-perfume, cola de sapateiro, chá de beladona (S17(35)A). Álcool, maconha, cocaína, haxixe, crack. (S17(35)D)

Efeitos eufóricos, calmantes, alucinógenos, depressores do sistema nervoso central (S6(48)A). Depressoras, perturbadoras, estimulantes. (S6(48)D)

Maconha te deixa com fome amarga, o crack só faz mal. (S18(22)A). Crack você fica [...] não vê o que você faz. $(S 18(22) D)$

Aumentou número de definições/classificações

Maconha, cocaína, base, crack, tabaco, álcool, heroína, dose, bala, LSD (S20(29)A).

Crack, cocaína, LSD, êxtase, maconha, álcool, cogumelo, roupinol, haxixe, beladona. (S20(29)D) 
Tenho alguns [...] (S5(35)A). Maconha, fome, sono, cocaína, crack perco o sono, arritmia cardíaca, alucinações. $(S 5(35) D)$

Cocaína: insônia, ansiedade. Maconha: depressoras, apático (S02(23)A). Ecstasy, cogumelos, maconha: perturbadoras. Anfetamina, cocaína: estimulantes. Álcool, sedativo, opioides: depressoras. (S02(23)D)

Heroína: depressora do sistema nervoso central. Diazepan (valium): calmante, ansiolítico (S06(48)A). Depressoras: álcool, opioides, codeína. Perturbadoras: maconha, LSD, cogumelos. Estimulantes: crack, cocaína, merla, anfetaminas. (S06(48)D)

Meu problema: cocaína e ecstasy, causava graves problemas físico e familiar (S19(24)A). Cocaína: inquietação. Maconha: tranquilidade. (S19(24)D)

Não S24(35). Álcool altera mente e comportamento. (S24(35)D)

Maconha [...] fico tranquilo, depois [...] vem preguiça. (S12(24)A). Causa euforia, depressão, angústia, desespero, falta de controle, perda familiar. (S12(24)D)

\section{Categoria 2 - Conhecimento correlacionado/contextualizado}

Mania de perseguição (S04(40)A). Eu não durmo diariamente. (S04(40)D)

Cocaína: euforia, inibidora de desejo sexual; Crack: irritabilidade, aumentadora do desejo sexual; Maconha: excitabilidade inicial e fome no final (S06(48)A). Cocaína: euforia, falta de apetite, paranóico. Maconha: euforia inicial, chapadão, fome. Crack: compulsão, irritabilidade, libido exacerbada, desconforto total. (S06(48)D)

Maconha fico alegre, dá fome. Crack fico assustado [...] querendo fumar mais para sentir a onda que é muito boa (S17(35)A). Crack fico assustado, suando [...] A maioria de nós adictos gosta de crack. (S17(35) D)

Quando eu usava crack, ficava assustado, com medo [...] (S23(28)A). Quando eu bebia, tornava agressivo. (S23(28)D)

Construir centros de recuperação com verbas do governo, aulas de literatura de NA e terapias, incentivo a esportes (S15(43)A). Aulas semanais nas escolas de ensino médio sobre o assunto. (S15(43)D)

Recomendaria uma casa de recuperação (S18(22)A). Honestidade. (S18(22)D)

Controlar o uso compulsivo, consultar um médico psiquiatra para uso de medicamentos ansiolíticos e insônica, participar do NA ou AA (S26(22)A). Acredito hoje na eficácia das intervenções voluntárias e involuntárias. (S26(22)D)

Correlacionou com maior clareza

Maconha: fome; crack: sem sono. (S08(26)A). O crack me deixava agitado ligado, sem controle. Maconha [...] sono e fome. $(S 08(26) D)$

Não (S15(43)A). Álcool: ficava muito nervoso, agressivo. Crack: ficava muito paranoico, cheio de pânico. Maconha me deixava tranquilo, preguiçoso. (S15(43)D)

Isolamento por usar a cocaína (S27(22)A). [...] meu comportamento fica totalmente descontrolado, com raiva, sem paciência, sem sentimento algum, e após vem a depressão. (S27(22)D)

Fazer a programação e procurar o poder superior [...] Deus (S5(35)A). Evitar velhos amigos, locais e hábitos de adicção. (S5(35)D)

[...] vamos à Igreja buscar Deus porque as drogas [...] vai acabar com sua vida e de sua família (S8(26)A). Palestras como esta que tivemos ou grupo com o NA, AA. (S8(26)D)

O ego é responsável pela voz do pensamento, logo quando se manifestar é só repetir "saudável"; de 1'; a 5';. [...] Substituição mental ou física (S10(38)A). Não espancar ou medicar simplesmente, usar 
psicologia [...] informações não maçantes, descontraídas [...]. (S10(38)D)

Conscientização, esclarecimento, que Deus nos ama, somos templo do espírito, intervenção medicamentosa (S22(34)A). Através de simpósios [...] estudos como esse da universidade (grupo de intervenção), terapias, programas e princípios. (S22(34)D)

Legenda:

*S: Participante

**(): Idade do participante

***A: Antes da Intervenção

****D: Depois da Intervenção

NA: Narcóticos anônimos.

A categoria 1 mostrou que os sujeitos ampliaram seu espectro de conceito sobre substâncias psicoativas, incluindo em suas definições mais substâncias lícitas, como os medicamentos. Na categoria 2, desenvolveram falas com maior pertinência em relação aos efeitos e ações psicoativas das substâncias no organismo, quando comparados às respostas antes das intervenções. Também aprofundaram a correlação/contextualização entre o comportamento percebido e o efeito da droga, auxiliando na elaboração de novas estratégias de reabilitação.

\section{DISCUSSÃO}

Em relação ao gênero abordado nesta investigação, os homens são mais propensos em relação às mulheres ao uso de drogas ilícitas ${ }^{(1,6)}$, além de outras variáveis, como baixo nível educacional e a inserção em um grupo de amigos em que a maioria faz uso de álcool, tabaco ou maconha ${ }^{(1)}$. Os aspectos de baixa escolaridade são indicados como desvantagem social e uso de drogas ilícitas ${ }^{(6)}$.

O resultado da pesquisa inova ao apontar o conhecimento constituído por pessoas que abusam de álcool e outras drogas acerca de aspectos que podem contribuir para a autonomia, ou seja, compreensão sobre o uso de drogas e a gestão dos próprios problemas decorrentes deste hábito. O principal foco foi compreender a educação em saúde como produção do conhecimento, e promoção do autocuidado, com o empoderamento no processo de cuidar. Durante os processos grupais, trocam-se conhecimentos e busca-se a compreensão dos prejuízos que o uso de substâncias químicas provoca à saúde ${ }^{(14)}$. Trata-se de um processo social de empoderamento do indivíduo, no exercício do pensamento reflexivo e crítico, na escolha e em decisões a respeito de sua própria saúde, amparado por profissional de saúde ${ }^{(7)}$.

O reconhecimento de tipos de substâncias já era familiar aos participantes, o que representou o saber prévio à intervenção obtido pela fase de perscrutação ${ }^{(12)}$, que não pode ser desconsiderada no processo de educação. Isto possibilitou a reflexão sobre os efeitos das substâncias psicoativas na vida de cada um e os levou às expressões de definições mais abrangentes. Tal efeito corrobora com um dos eixos de autonomia, que é a compreensão do uso de substâncias psicoativas e de seus efeitos ${ }^{(15)}$.

No mesmo sentido, a reelaboração da classificação e ação de substâncias por parte dos sujeitos da pesquisa agregará fatores de proteção a uma população que apresenta característica de uso de múltiplas drogas. Um padrão de abuso de drogas concomitantes entre o álcool, tabaco, maconha, ecstasy e derivados de anfetamina ${ }^{(1,3)}$ e medicamentos ${ }^{(2)}$ são associações que aumentam substancialmente os riscos e danos à saúde, sobretudo quando somado à desvantagem social(16), condição semelhante às dos sujeitos do estudo.

O reconhecimento de substâncias de abuso inicia-se com a abordagem do tabaco, uma vez que a maioria dos sujeitos usuários de substâncias ilícitas são fumantes, o que pode ser compreendido pelo fato de a nicotina ser um fator importante na progressão do uso de drogas para a dependência ${ }^{(17)}$. Isto é, cada vez mais, tem-se o uso do tabaco associado com drogas como álcool e maconha ${ }^{(6,17)}$.

Na pesquisa, os estimulantes, como a metanfetamina e a cocaína, foram utilizados em situações de enfrentamento de tarefas que exigiam longas horas de trabalho e agilidade. Maconha, alucinógenos 
e drogas de prescrição foram escolhidos pelos efeitos de relaxamento. Conhecido o resultado das substâncias, os usuários passam a gerenciar a sinergia entre elas, tal como reduzir o pico das drogas excitatórias, consumindo a maconha para diminuir o pico de ecstasy no organismo ${ }^{(15)}$.

Nas práticas educativas nesta pesquisa foi frequentemente abordada a correlação de comportamento e a busca incessante da droga. Hábito que pode ser explicado em, parte, por experiências com substâncias químicas que afetam circuitos regulatórios da recompensa, motivação, memória e tomada de decisão ${ }^{(4)}$. Os fatores de dependência das drogas são estudados perante sua ação de gratificação no circuito límbico-córtico-estriado(4).

A partir desta compreensão, os sujeitos da pesquisa iniciaram a revisitação do próprio comportamento compulsivo e crônico de abuso de drogas. Neste mesmo momento, aprofundaram e identificaram danos à saúde vivenciados, muito destes compatíveis com a literatura, tal como o uso crônico de drogas ilícitas causando danos neurológicos e déficit de aprendizagem, além de consequências psicológicas e sociais ${ }^{(4)}$.

Diante do relato dos sujeitos, entre as substâncias mais consumidas, há a cocaína, que promove rapidamente efeitos agradáveis e prazer, mas o uso compulsivo pode levar a diversidade de sintomas de origens neurobiológicas ${ }^{(4)}$. Já indivíduos que abusam do álcool apresentam pior desempenho na memória tardia ${ }^{(18)}$, bem como as variações de estado de humor, comportamento verbal e emocional, com impacto negativo nas interações sociais, sobretudo na presença de outro indivíduo, que também se encontra alcoolizado ${ }^{(5)}$.

O processo de educação em saúde que os sujeitos deste estudo vivenciaram também permitiu a ampliação da identificação das drogas psicoativas prescritas. Nos Estados Unidos, o uso de drogas psicoativas prescritas é visto como uma epidemia e um grave problema de saúde pública, mobilizando estratégias políticas e assistenciais com métodos de redução de danos e controle dos profissionais e dos serviços de saúde ${ }^{(19)}$.

Prosseguindo, acerca do conhecimento das estratégias de reabilitação, os sujeitos relataram a religião, a abordagem dos Narcóticos Anônimos (NA), a psicoterapia e a presença dos pesquisadores com o grupo de educação em saúde. Nessa conjunção, a crença religiosa pode ter uma implicação protetora sobre o uso e o abuso de substâncias psicoativas ${ }^{(20)}$, com melhoria da saúde física e mental, além de proteger usuários que estão em tratamento e reabilitação de uma recaída, com relevante papel na fase de recuperação ${ }^{(21)}$. Evidências indicam que o uso de drogas é baixo entre indivíduos com práticas religiosas regulares ${ }^{(22)}$.

Para as citações do NA, é importante ressaltar que as discussões científicas frequentemente são feitas lembrando-se também dos grupos de Alcoólicos Anônimos (AA) ${ }^{(3,22-24)}$. Trata-se de grupos de autoajuda na recuperação de pessoas, com fundamento no programa dos 12 passos no auxílio à recuperação. Autores referem que a espiritualidade é o principal elemento no tratamento, no qual os usuários são induzidos a confiarem em um poder superior que os conduz ao sentimento de negação da recaída, muito efetivo em diversos grupos, sobretudo em mulheres, adolescentes e moradores urbanos ${ }^{(23-24)}$.

Há referência de que o tratamento combinado, utilizando educação, psicoterapia e medicação, é desejado e assertivo. Indivíduos que fazem uso e abuso de substâncias psicoativas têm maior probabilidade de desenvolver algum transtorno mental ${ }^{(25)}$. Quando apresentam comorbidade, como transtornos mentais, a psicoterapia tem demonstrado resultados positivos, quando combinada com a farmacoterapia ${ }^{(26)}$.

Os estudos mostram que a intervenção educativa mobiliza melhoria dos comportamentos de saúde da população que recebe o cuidado ${ }^{(27)}$, ou seja, o processo educativo promove a saúde das pessoas, e o uso da psicoeducação visa à adesão ao tratamento e à redução de recaídas, até mesmo nas situações de comorbidade com transtornos mentais graves ${ }^{(10-11)}$.

Quanto ao trabalho do enfermeiro, com população que abusa de substâncias, há de se superar os sentimentos de despreparo e insegurança, ou a visão negativa em relação à condição de dependência e aos problemas advindos dessa situação. Contudo, reconhece-se a importância de ações educativas abrangendo não só profissionais, mas familiares e usuários, no sentido de melhorar e ampliar a atenção à saúde ${ }^{(28)}$. Além do mais, a família deve ser incluída, pois o uso e o abuso de substância psicoativa 
afetam o indivíduo, a família e o meio social em que está inserido ${ }^{(29)}$.

Resultados de estudo realizado em comunidade terapêutica, com enfermeiros que cuidavam de pessoas que conviviam com HIV/AIDS, desvelaram a relevância da instrução em saúde mental para a efetivação do trabalho. Aplicar esses conhecimentos em processos de educação e promoção da saúde das pessoas em abuso de álcool e outras drogas, ou que possuem sofrimento mental, contribuiu positivamente com o tratamento, levando conforto e auxiliando na melhor compreensão do seu estado de saúde e prevenindo crises $^{(8)}$.

\section{CONCLUSÃO}

Observou-se a edificação de conhecimento dos saberes primário quanto ao uso de drogas, em destaque a correlação com o próprio comportamento e a contextualização na busca pela reabilitação. A análise instruída pela pesquisa convergente assistencial possibilitou a identificação do conhecimento prévio dos tipos de drogas que os sujeitos constituíram por meio da própria vivência. Isso corroborou para a construção de conhecimento de outros temas relacionados, como a melhor qualidade e profundidade do saber sobre os efeitos das substâncias, sua nocividade à saúde e à vida social e os caminhos possíveis para reabilitação.

Ao considerar esses resultados, sugerem-se contribuições para modelos de intervenção e cuidados em enfermagem. Primeiro, considerar o conhecimento que cada sujeito traz na bagagem da vida, segundo, reconhecer o fenômeno do uso concomitante de múltiplas drogas e sua gravidade para estratégias de redução e danos e, terceiro, evidenciar os danos mentais, neurológicos e cognitivos que o consumo crônico de drogas pode ocasionar nos sujeitos usuários.

Por fim, destaca-se a consistência da constituição de autonomia, apontadas nas reflexões dos sujeitos acerca dos efeitos das substâncias utilizadas com o próprio comportamento, levando-os a se perceberem e correlacionarem o conhecimento construído. Tanto quanto a compreensão de que, para este problema tão complexo, as estratégias de reabilitação devem ser diversas e em variados âmbitos da sociedade (religioso, terapêutico, organizações sociais, educação em saúde).

Admite-se como limitação desta intervenção os resultados medianos da análise quantitativa, o que sugere desenvolvimento de instrumentos mais precisos e ajustados em relação à concepção da educação em saúde, que por sua vez aborda dimensões que não são facilmente mensuráveis por indicadores quantitativos. Não obstante, a educação em saúde demonstrou-se um caminho assertivo ao proporcionar caminhos para o empoderamento e a corresponsabilidade desejáveis na promoção, reabilitação e prevenção de agravos da saúde.

\section{AGRADECIMENTO}

À Agência de Fomento à Pesquisa Fundação de Amparo à Pesquisa do Estado de Goiás (FAPEG), edital 06 de 2012.

\section{REFERÊNCIAS}

1. Quek LH, Chan GC, White A, Connor JP, Baker PJ, Saunders JB, et al. Concurrent and simultaneous polydrug use: latent class analysis of an Australian nationally representative sample of young adults. Front Public Health. 2013;1(61):1-9.

2. Kelly BC, Wells BE, Pawson M, LeClair A, Parsons JT. Combinations of prescription drug misuse and illicit drugs among young adults. Addict Behav. 2014;39(5):941-4.

3. Kelly AB, Chan GC, White A, Saunders JB, Baker PJ, Connor JP. Is there any evidence of changes in patterns of concurrent drug use among young Australians 18-29 years between 2007 and 2010? Addict Behav. 2014;39(8):124952. 
4. Cadet JL, Bisagno V, Milroy CM. Neuropathology of substance use disorders. Acta Neuropathol. 2014;127(1):91107.

5. Kirkpatrick MG, deWit H. In the company of others: social factors alter acutealcohol effects. Psychopharmacology (Berl). 2013;230(2):215-26.

6. Secades-Villa R, Garcia-Rodríguez O, Jin CJ, Wang S, Blanco C. Probability and predictors of the cannabis gateway effect: a national study. Int J Drug Policy. 2015;26(2):135-42.

7. Monteiro EMLM, Vieira NFC. Health education based on culture circles. Rev. bras. enferm. 2010;63(3):397-403.

8. Allen J, Hamilton A, Nunn R, Crock L, Frecker J, Burk N. Evaluation of a community-based mental health drug and alcohol nurse in the care of people living with HIV/AIDS. J Psychiatr Ment Health Nurs. 2009;16(2):129-36.

9. Valença CN, Brandão ICA, Germano RM, de Vilar RLA, Monteiro AI. Abordagem da dependência de substâncias psicoativas na adolescência: reflexão ética para a enfermagem. Esc. Anna Nery. 2013;17(3):562-7.

10. Rummel-Kluge C, Kluge M, Kissling W. Frequency and relevance of psychoeducation in psychiatric diagnoses: Results of two surveys five years apart in German-speaking European countries. BMC Psychiatry. 2013;13(170):1-5.

11. Petretto DR, Preti A, Zuddas C, Veltro F, Rocchi MB, Sisti D, et al. Study on psychoeducation enhancing results of adherence in patients with schizophrenia (SPERA-S): study protocol for a randomized controlled trial. Trials. 2013;14(323):1-13.

12. Trentini M, Paim L. Pesquisa convergente-assistencial: um desenho que une o fazer e o pensar na prática assistencial de saúde-enfermagem. $2^{\text {a }}$ ed. Florianópolis: Insular; 2004.

13. Ministério da Saúde (BR). Conselho Nacional de Saúde. Diretrizes e normas regulamentadoras de pesquisas envolvendo seres humanos. Resolução n. 466, de 12 de dezembro de 2012. Brasília; 2012.

14. Vasconcelos SC, Frazão IS, Ramos VP. Grupo terapêutico educação em saúde: subsídios para a promoção do autocuidado de usuários de substâncias psicoativas. Cogitare Enferm. 2012;17(3):498-505.

15. Boeri M, Sterk C, Bahora M, Elifson K. Poly-Drug Use among Ecstasy Users: Separate, Synergistic, and Indiscriminate Patterns. J Drug Issues. 2008;38(2):517-41.

16. Daniel JZ, Hickman M, Macleod J, Wiles N, Lingford-Hughes A, Farrell M, et al. Is socioeconomic status in early life associated with drug use? A systematic review of the evidence. Drug Alcohol Rev. 2009;28(2):142-53.

17. Huang YY, Kandel DB, Kandel ER, Levine A. Nicotine primes the effect of cocaine on the induction of LTP in the amygdala. Neuropharmacology. 2013;(74):126-34.

18. Wetherill RR, Fromme K. Acute alcohol effects on narrative recall and contextual memory: An examination of fragmentary blackouts. Addict Behav. 2011;36(8):886-9.

19. Winstanley EL, Gay J, Roberts L, Moseley J, Hall O, Beeghly BC, et al. Prescription drug abuse as a public health problem in Ohio: a case report. Public Health Nurs. 2012;29(6):553-62.

20. Moscati A, Mezuk B. Losing faith and finding religion: Religiosity over the life course and substance use and abuse. Drug Alcohol Depend. 2014;(136):127-34.

21. Pezzella FS, Vlahos S. The moderating influence of religion on the behavioral health of formerly incarcerated men. J. Relig. Health. 2014;53(6):1873-84.

22. Al-Omari H, Hamed R, Abu Tariah H. The Role of Religion in the Recovery from Alcohol and Substance Abuse Among Jordanian Adults. J Relig Health. 2015;54(4):1268-77.

23. Laudet $\mathrm{AB}$. The impact of alcoholics anonymous on other substance abuse related twelve step programs recent dev alcohol. Recent Dev Alcohol. 2008;(18):71-89.

24. Krentzman AR, Robinson EAR, Moore BC, Kelly JF, Laudet AB, White WL, et al. How Alcoholics Anonymous (AA) and Narcotics Anonymous (NA) work: cross-disciplinary perspectives. Alcohol Treat Q. 2010;29(1):75-84. 
25. Fard JH, Gorji MA, Jannati Y, Golikhatir I, Bozorgi F, Mohammadpour R, et al. Substance dependence and mental health in northern Iran. Ann Afr Med. 2014;13(3):114-8.

26. Kelly TM, Daley DC, Douaihy AB. Treatment of substance abusing patients with comorbid psychiatric disorders. Addict Behav. 2012;37(1):11-24.

27. Kara B. The efficacy of an educational intervention on health behaviors in a sample of Turkish female nursing students: A longitudinal, quasi-experimental study. Nurse Educ. Today. 2015;35(1):146-51.

28. Neville K, Roan N. Challenges in Nursing Practice Nurses' Perceptions in Caring for Hospitalized MedicalSurgical Patients With Substance Abuse/Dependence. J Nurs Adm. 2014;44(6):339-46.

29. Simsek N. Nursing care of individuals and their families with substance use disorders. J Psy Nurs. 2010:96. 